\title{
Co-Designing a Recommender System for the Elderly
}

\author{
Madita Herpich \\ Hochschule Augsburg \\ Augsburg, Germany \\ madita.herpich@informatik. \\ uni-augsburg.de
}

\author{
Thomas Rist \\ Hochschule Augsburg \\ Augsburg, Germany \\ thomas.rist@hs- \\ augsburg.de
}

\author{
Elisabeth André \\ Universität Augsburg \\ Augsburg, Germany \\ andre@informatik.uni- \\ augsburg.de
}

\begin{abstract}
To promote a healthy life-style and to increase well-being of elderly people, we have extended a digital picture frame - the CARE system - that interleaves a picture display mode with a recommender mode. In recommender mode, CARE encourages its users to engage in physical and mental training activities. The success of such a system essentially depends on the extent to which given recommendations are followed. The current paper investigates in how far rewarding schemes as known from computer games could be deployed in order to increase user appreciation of the CARE system. To this end, we prepared and ran a co-design workshop with a peergroup of senior citizens. Finally, we draw conclusions that will guide our work towards a gamified version of CARE.
\end{abstract}

\section{CCS Concepts}

- Human-centered computing $\rightarrow$ Human computer interaction;

\section{Keywords}

Technologies for improving quality of daily living, co-design practices with elderly

\section{INTRODUCTION}

The project CARE (Context-Aware Recommender System for the Elderly [7],[3]) addresses the question of how information technologies could increase and support the live quality of solitary living elderly people. The project augmented a digital picture frame with a recommender mode. In picture frame mode, the CARE system retrieves pictures (including various motives, such as landscapes, flowers, pets etc.) and photographs of family members for display (see Fig. 1). When switched to recommender mode, CARE provides hints on activities that potentially contribute to increase the user's well-being. For instance, CARE may suggest physical or mental activities, or give hints on how to increase comfort and ease of the living environment. For each

This is the author's version of the work. It is posted here for your personal use. Not for redistribution. The definitive Version of Record was published in:

PETRA '17, June 21-23, 2017, Island of Rhodes, Greece

(C) 2017 ACM. ISBN 978-1-4503-5227-7

DOI: http://dx.doi.org/10.1145/3056540.3064948 category, a range of prepared recommendations is available in a content repository. For instance, recommendations for physical activities comprise physical exercises for training motor skills, balance, and strength.

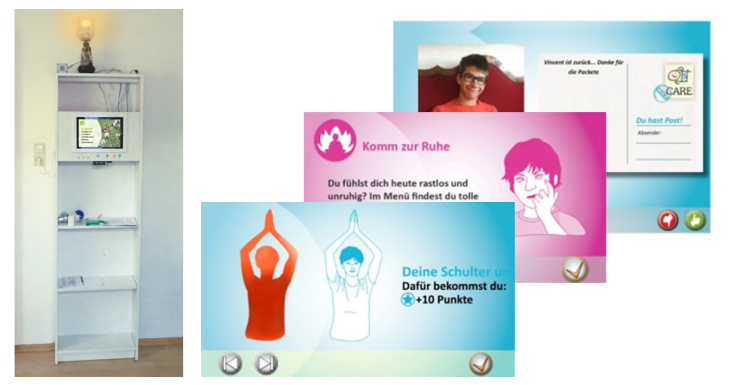

Figure 1: CARE prototype with sample screens

Whether a system like CARE can reach its aims essentially depends on whether the given recommendations are followed in general and - depending on the kind of recommendation - how accurately they are followed. For example, it is crucial that the body movements of a recommended physical exercise are performed as instructed. Also, as CARE has been designed for long term usage, users need to be encouraged to follow repeatedly recommended activities. With the ambition to increase the effectiveness of CARE, it was questioned, in what way well-known computer game elements and principles, such as live feedback and especially rewards, may be used to enhance the user's overall appreciation of the CARE system. However, augmenting CARE in such a direction raises a number of questions:

- Q1: Which activities recommended by CARE should get rewarded?

- Q2: What kind of rewards would be appropriate?

- Q3: Should CARE come with a uniform rewarding scheme for all recommended activities?

\section{RELATED WORK}

Following Deterding et al. [2], gamification is referred to as "the use of game design elements in non-game contexts". A prominent class of such game design elements are all kinds of rewarding scheme. Wang et al. [9] distinguish between eight different forms of rewards: "Score systems", "Experience point reward systems", "(Virtual) Item granting systems", "(Virtual) Resources", "Achievement systems" 
referred to "titles", "Feedback messages", "Plot animations and pictures", "Unlocking mechanisms" or "Access".

Of high relevance for our work on the CARE system are studies and projects that address gamification to promote a healthier lifestyle and to increase overall well-being Lumsden et al. [5] reviewed 33 studies on gamified applications for cognitive training and assessment purposes. They identified a number of different reasons for deploying game elements in cognitive training and testing, including the aim to increase the users' motivation and long-term engagement, as well as usability, intuitiveness and appeal of an application. In a more recent literature research Johnson et al. [4] revisited 19 papers that report empirical evidence on the effect of gamification on health and wellbeing including both physical as well as mental exercises. While the majority of studies reported positive effects, about 41 per cent reported mixed or neutral effects especially with regard to cognitive outcomes.

Meta studies provide a good link to cases of successful and less successful gamification approaches. However, they do not explain why gamification worked or failed. In order to better understand how game design elements motivate user behavior in non-game contexts, several attempts have been made to discuss the role of incentives and rewards as found in computer games in the light of psychological theory. Such an approach is presented by Richter et al. [6]. Important to their analysis is Self-Determination Theory [8] that distinguishes between intrinsically motivated behaviours, which are performed out of interest and satisfy the innate psychological needs for competence and autonomy, and extrinsically motivated behaviours, which support activities (e.g., collecting points) that are done in order to attain some outcome separable from the primary task (e.g., physical exercising). Richter et al. consider the use of points, badges, leaderborads etc. as applications of extrinsic motivators. They conclude that careful selection and implementation of extrinsic motivators will trigger internal motivation and aid in maintaining it. However, they point out that success in one non-game context does not guarantee that the same mechanism will be successful in another non-game context.

While the majority of studies suggest that gamification has worked in many cases, it still remains a challenge for application designers to decide on which gamifying elements should be added because they are likely to add value to an application, and which one should be neglected since they may even cause opposite effects. Suggestions for application design can be extracted from works compiling gamification guidelines based on interviews with seniors [1].

\section{METHOD: CO-DESIGN WORKSHOP}

\subsection{Objectives}

We conducted a workshop with elderly people to pursue the following objectives:

1. to learn about the daily routines of the participants and whether they already engage in activities that promote a healthy lifestyle,

2. to elicit feedback on technology that actively gives recommendations and suggest activities,

3. to gather opinions on what could establish a meaningful rewarding scheme for recommended activities

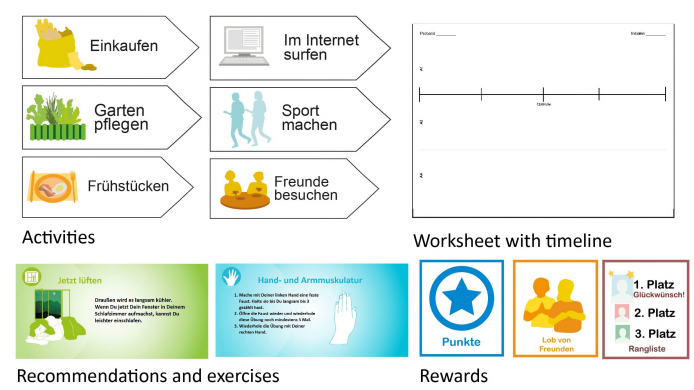

Figure 2: Pre-prepared workshop materials

\subsection{Preparation}

\section{Participants and location.}

We contacted a local seniors' association and it was agreed that we could meet a group of about 10-15 retired seniors ageing between 60 and 70 at their weekly gathering, all willing to take part in our workshop.

\section{Worksheet and Cards.}

To elicit information about the participants' daily routines and activities, and to reveal their attitudes towards rewarding schemes, a working sheet with a timeline and three piles of cards has been prepared, see Fig. 2. The first pile consisted of cards depicting daily routines. Cards of the second pile showed activities that CARE would recommend to promote a healthier lifestyle, e.g., physical or mental exercises. By means of cards from the third pile, participants could allocate different kinds of reward to activities. While there is a huge variety of possible rewards, we focused on three types of reward, which we found suitable for CARE: scores, badges and virtual currencies. These again were transferred into different subcategories (see Fig. 3). The category scores encompasses rewarding in form of comparison with one self, like a high-score in speed races, and with other players in a leaderboard. Badges are a means to represent the status of each player (CARE user) by providing him or her with awards or the recognition by others. The virtual currency category stands for all kind of rewards which can be exchanged with virtual or real goods. For instance, virtual coins may be used to purchase yet needed pieces of a jigsaw puzzle on screen or to have a virtual plant grow and prosper. Another option would be to use the coins for purchasing real-world goods, such as a cup of coffee. Finally, a number of blank cards were included so that participants could use them to write down additional activities or rewards for which no cards had been prepared. With exception of blank cards, the visual appearance of all cards resembled the graphical style used for screen content displayed by the CARE system.

\section{Predefined questions for semi-structured interviews.}

Semi-structured interviews were prepared in which participants would be involved at several stages of the workshop to receive feedback on a number of questions, such as whether participants already care about a healthy lifestyle and how this is mirrored in their daily routines, what kind of reward schemes participants are already familiar with, or what they think about a recommendation system like CARE. 


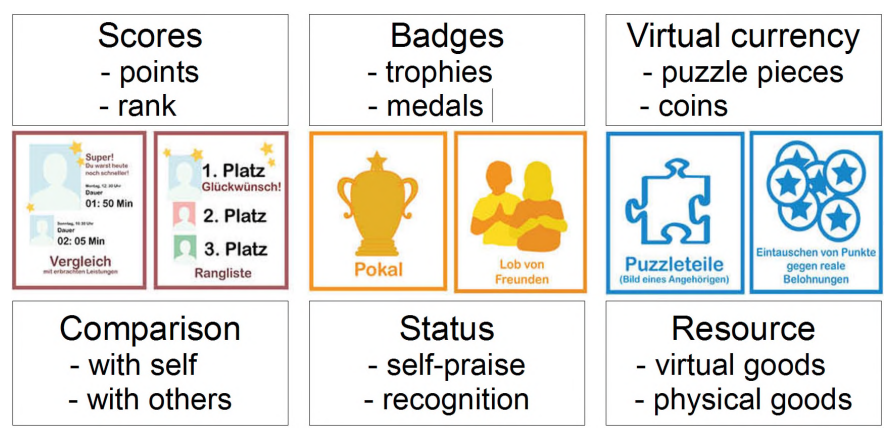

Figure 3: Reward options in CARE

\subsection{Procedure}

The workshop took place at a senior meeting centre. 11 women and one man of different age between 60 and 70 participated actively in the workshop. The workshop was administrated by one female and two male experimenters. In the workshop, participants:

- received an introduction to the CARE project and the existing CARE prototype including the opportunity to try-out practising instructed and monitored by the CARE prototype,

- got an explanation of their role in helping us extend and improve the CARE system,

- took part in three subsequent card-laying tasks to give us an idea about their daily routines and activities, and to reveal their attitudes towards rewarding schemes,

- were asked to provide verbal feedback on a number of predefined questions which are to be prompted by the experimenters during the workshop.

Throughout the workshop the three experimenters performed data gathering by taking notes on verbal responses and comments, and by taking pictures of the outcomes of the cardlaying tasks. Grouping and analysis of the recorded material took place after the workshop.

For the first task, prepared worksheets were distributed and the task was explained (see Fig. 2). Participants were requested to give an outline of their individual daily routine by picking suitable activity cards and arranging them on the timeline. To make them reflect on current exercise habits, it was noted that there are activity cards depicting physical or mental exercises, and that they could use those cards as well. The experimenters also answered questions on cards in case their meaning required further explanation. Participants were also told that they could use blank cards to include activities not depicted on pre-prepared cards. Completion of this task took about 20 minutes. Participants were requested to keep their worksheet as they would shortly need it for a further task.

For the second task, participants were asked to look at their individual daily routines and indicate what kind of recommendations as those given by CARE (such as physical exercises or quizzes) they would like to receive, and at what time during the day. They could do so by inserting cards representing recommendations on their working sheets. Again, the experimenters helped clarify questions regarding recommendation cards and the task itself. As a result, all participants augmented their worksheet configurations by at least some recommended activities. This task was completed within 20 minutes.

Next, a group discussion was stimulated to elicit the kinds of rewarding system that they knew. Mentioned items were noted by the experimenters. The discussion was continued by introducing scores, badges, and virtual currencies as possible rewards for activities. After a common understanding of these concepts was achieved, the participants were asked to review all activities on their worksheets and think about whether they would like to receive a reward in case of an activity has been done, and if so, what kind of reward they would consider appropriate. Participants were told that they can easily establish an association between an activity and a reward by picking a reward card and putting it closely to a target activity card on their worksheets. Completing this task took about 30 minutes. For later analysis, pictures were taken of all final worksheet configurations (see Fig. 4).

\subsection{Outcomes and Observations}

\section{Task 1: Daily routines.}

All participants described their daily lives by arranging activity cards on a timeline, starting with getting up in the morning and finishing with going to sleep in the evening. As all but one participant used to have lunch around noon, daily routines could be naturally structured into morning (prelunch) activities and after-lunch activities. As expected, a comparison of the resulting worksheets revealed moderate variations in both number and kind of mentioned activities from one participant to the other. On the other hand, there was a strong pattern regarding the timing of some activities. For instance, all participants indicated cleaning and shopping being part of their agendas but typically before lunch (for cleaning in 12/12 and, for shopping in 11/12 cases).

In view of CARE we were in particular interested in activities that potentially contribute to a healthier lifestyle and increased well being. To this end, we counted the number of such activities on the agendas and found that before lunch only doing sports (7/12) fits into this category. In contrast, after lunch all participants indicated at least 2, and in one case even 5 such activities (including take a nap, go out for a stroll, visit friends, do sports). Some participants included the "hobby" card into their agendas to refer to hobby-related activities that were not covered by prepared cards. None of the participants had a need to use a blank card for routine activities not yet depicted on pre-prepared cards.

\section{Task2: Appreciated recommendations.}

In response to Task2, all participants augmented their agendas by recommendations for activities as they could be provided by CARE. We observed great variations in number (between 2 and 8, average 4) and kind of wanted recommendations. In the morning, recommendations for airing the room $(9 / 12)$ and for doing sports $(7 / 12)$ were most frequently mentioned, while for the second half of the day recommendations for relaxing scored highest (9/12). To our surprise, none of the participants wanted to receive recommendations related to social activities, such as visiting friends. We also looked whether selected recommendations were related to activities already included in a participant's agenda (cf. Task1). This was important to see whether a recommendation would either serve as a reminder to do 
something that one usually does, or as a stimulation to do something in addition to daily routines. In case of recommending physical exercising those who already do sports would appreciate reminders to carry on, and they are also open to receive stimulation for other physical exercises. Recommendations of relaxation-related activities were classified as "stimulators" as none of such actives were included in any agenda after competition of Task1.
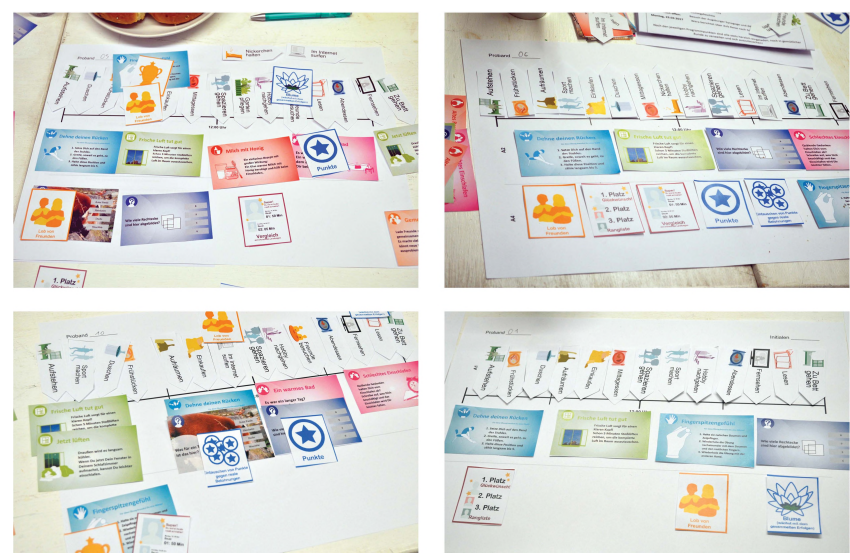

Figure 4: Examples for finished worksheets

\section{Task3: Rewards for activities.}

Regarding reward schemes that participants already knew, some referred to their times in school where they earned sport badges, stickers or achievement stamps. Most were also familiar with commercial rewarding schemes, such as Pay Back cards handed out by some super market chains. Further, known types of rewards were praise by friends for having cooked a good meal for them, or just receiving a phone call after a nice visit of a friend or family member. One participant felt rewarded when her grandchildren got good marks in school because she helped them with doing their homework. Also, there was common agreement among the workshop participants that it is often the activity itself or its outcome that constitutes a tangible reward to them.

Examining worksheets again to see which activities got associated with rewards revealed a quite heterogeneous picture. One participant did not assign any reward arguing that having accomplished an activity is enough rewarding itself and doesn't need an extra reward on top of it. In three cases participants rewarded only activities which were already part of their agendas (such as cleaning, gardening, or surfing the Internet as this was considered a challenging activity) but not to recommended activities (cf. Task 2). In contrast, seven participants only rewarded recommended activities, and only one participant rewarded both, routine activities as well as recommended activities. With regard to reward types we observed a strong preference (21 cases) for virtual currencies that could be used to purchase real or virtual goods. Several suggestions were given for potential exchanges, such as using collected virtual currency for sports equipment. Badges were assigned in 6 cases, and scores in another 5 cases. However, only three participants rewarded activities consistently with the same type of reward, while all others suggested different reward types for different activities. Also, we did not observe dominant occurrences of activity/reward associations which indicates different individual preferences across the workshop participants.

\section{CONCLUSIONS FOR A GAMIFIED VER- SION OF CARE}

The outcomes of our co-design workshop are meant to help us in making design decision on how to gamify CARE. Regarding our initial questions (Q1, Q2, Q3) we feel encouraged to incorporate a reward mechanism into CARE. However, we no longer think that a uniform rewarding scheme across all recommendation types is the approach of choice. Rather, we plan a gamified CARE version that allows its users to configure the rewarding schemes individually. In configuration mode, users should be enabled to easily select activities and associate them with reward types (Q3) that they find suitable.

\section{ACKNOWLEDGMENTS}

We would like to thank A. Seiderer, S. Klapprott, the ASB Göggingen, Augsburg, the members of the "Mehrgenerationstreffen" and E. Verdura of the ASB Göggingen.

\section{REFERENCES}

[1] B. De Schutter and V. Vanden Abeele. Designing meaningful play within the psycho-social context of older adults. In Fun and Games '10, pages 84-93, New York, NY, USA, 2010. ACM.

[2] S. Deterding, D. Dixon, R. Khaled, and L. Nacke. From game design elements to gamefulness: Defining "gamification". In MindTrek '11, pages 9-15, New York, NY, USA, 2011. ACM.

[3] S. Hammer, A. Seiderer, E. André, T. Rist, S. Kastrinaki, C. Hondrou, A. Raouzaiou, K. Karpouzis, and S. Kollias. Design of a lifestyle recommender system for the elderly: Requirement gatherings in germany and greece. In PETRA '15, pages 80:1-80:8, New York, NY, USA, 2015. ACM.

[4] D. Johnson, S. Deterding, K.-A. Kuhn, A. Staneva, S. Stoyanov, and L. Hides. Gamification for health and wellbeing: A systematic review of the literature. Internet Interventions, 6:89-106, 2016.

[5] J. Lumsden, E. A. Edwards, N. S. Lawrence, D. Coyle, and M. R. Munafò. Gamification of cognitive assessment and cognitive training: a systematic review of applications and efficacy. JMIR Serious Games, 4(2), 2016.

[6] G. Richter, D. R. Raban, and S. Rafaeli. Studying gamification: The effect of rewards and incentives on motivation. In T. Reiners and L. C. Wood, editors, Gamification in Education and Business, pages 21-46, Cham, 2015. Springer International Publishing.

[7] T. Rist, A. Seiderer, S. Hammer, M. Mayr, and E. André. Care: Extending a digital picture frame with a recommender mode to enhance well-being of elderly people. In PervasiveHealth '15, pages 112-120, 2015.

[8] R. M. Ryan and E. L. Deci. Intrinsic and extrinsic motivations: Classic definitions and new directions. Contemporary educational psychology, 25(1):54-67, 2000.

[9] H. Wang and C. Sun. Game reward systems: Gaming experiences and social meanings. In DIGRA 2011, 2011. 